\title{
Vehicle To Vehicle Communication Using Li-Fi Technology
}

\author{
Arun Kumar $\mathbf{N}^{1}$, Hari Subramanian $\mathbf{M}^{2}$, Anurudh $\mathbf{H}^{3}$, Ms.M.Jaishree ${ }^{4}$ \\ Bachelors in Engineering, Sri Krishna College of Technology, Coimbatore ${ }^{1,2,3}$ \\ Assistant Professor, Electronics and Communication, Sri Krishna College of Technology, Coimbatore ${ }^{4}$
}

\begin{abstract}
Vehicle to Vehicle communication is the communication methodology which implies the network-based topology. This proposed method meets and ensures the comfort and safety for the vehicles by preventing major accidents. This proposed project is an application of vehicle to vehicle and no infrastructure is required. Since this project does not require any protocols or any architecture which is network based, this is more robust. This proposed project makes use of various sensors namely accelerometer, ultra sonic sensor, speed sensor and transfers the data collected from these sensors between vehicles. Services such as warning about an accident, traffic jams information or messages about a rescue vehicle which is approaching. Additional information such as whether information and voice commands can also be given. Moreover the main key part in this proposed system is the use of Light Fidelity ( $\mathrm{Li}-\mathrm{Fi})$ to transmit the data. The use of $\mathrm{Li}-\mathrm{Fi}$ ensures and guarantee the speed of the communication and also go green to the environment.
\end{abstract}

Keywords: Vehicle to Vehicle, Li-Fi, LED.

\section{INTRODUCTION}

In the last century, radio was introduced and implemented and gave rise to the new wireless world. It will be very surprising to know that the first wireless transmission of voice was done by the help of light waves. In 1880 the scientist Alexender Grahem Bell discovered the first wireless voice communication with the range over a distance of $213 \mathrm{~m}$. this invention was one of the greatest invention for him but the invention of radio communication was given high priority and his invention was covered by radio communication. As mobile phone came across the world the radio communication became more popular and due to lots of mobile appeared to the face of public so the popularity was increased for the radio communication. Li-Fi is transmission of data through light by using fibre optics and sending data through a LED that varies in intensity. Faster than the human eye can follow. Integrated chips inside LED will do the processing and amplification of data. The light intensity can be manipulated to send data by very small changes in the results. The technology transfers Thousands of data simultaneously in higher speed with the help of special modulation and demodulation technique.

Li-Fi technology is high intensity brightness LED,,s. Light emitting diodes can be made to switch on and off faster since operating speed of LED,,s is even less than one $\mu \mathrm{s}$, than the human eye can detect, causing the light source to be appear continuously. This on-off activity cannot be seen with the naked eyes of the human and that enables a kind of data transmission using codes. Switching on and LED is a logic 1switch off is a logic 0 the data can be encoded from the light wave and the exact information can be achieved.

Modulation is so fast that human eye doesn't notice a light sensitive device receives the signal and converts it back into original data. This method of using the light waves and frequency in it and sending the required data refers as Visible Light Communication (VLC) though its potential to compete with conventional Wi-Fi has inspired the popular characteristics LiFi. Visible Light Communication $\mathrm{Li}-\mathrm{Fi}$ is one of the very efficient version of $\mathrm{Wi}-\mathrm{Fi}$, which is based on visible light communication (VLC). This Li-Fi uses light for data communications medium using visible light waves as optical carrier for data transmission and illumination.

In this previous work, the communication of intra-vehicle and $\mathrm{V} 2 \mathrm{~V}$ to enable the safety of the vehicle is used. Namely, it extracted the vehicle information by connecting the OBD system using Pad's Bluetooth interface. And a WIFI connection enables the Pad to present a GUI for anti-collision warning, which is based on an offline map to show the real time GPS information of vehicles. Furthermore, it used the system board which supported the IEEE 802.11p/1609.X protocols to make information exchanged in vehicles. And the LTE module enables a remote server to monitor all vehicles on the road. In this project, an application of Vehicle to Vehicle (V2V) communication which does not require any infrastructure is proposed. This methodology is more robust since this vehicle to vehicle communication method does not require any network-based architecture or protocols. 


\title{
International Journal of Advanced Research in Computer and Communication Engineering
}

\author{
Vol. 8, Issue 2, February 2019
}

Vehicle-to-vehicle communication can be used to distribute various messages of multiple actions which are generated by the vehicle by using various sensors present in the vehicle. Services such as warning about an accident, traffic jams information or messages about a rescue vehicle which is approaching. Moreover, information about the appearance of road or conditions about the weather can be also transmitted.

\section{LITERATURE REVIEW}

This section provides the basic significance of vanet in cloud. It also provides the numerous methodology to efficiency and privacy aware techniques. This web development has resulted in huge usage of many applications and other serviceoriented applications.

Secured Data Processing, Notification and Transmission in a Human-Vehicle Interaction System-. Project CASA (Car Safety Apps) is a joint project of a consortium composed of 5 industrial and one academic partners in France with an aim of building an Android app that promotes safe and green driving, and secured data transmission. In this paper, we focus on the security aspects and features of our project. We present that the multimodal data processing of our work is safe and secured.

Security and Privacy for Sensor Networks-. In this work, explore a variety of security and privacy challenges facing sensor networks, and then look at defines strategies that may be employed to protect against. These threats. Since the amount of threats is enormous, we will begin by providing high-level discussion of different attacks or solutions and then focus our discussion one specific sampling of topics.

Three Decades of Driver Assistance Systems: Review and Future Perspectives- This contribution provides a review of fundamental goals, development and future perspectives of driver assistance systems. Mobility is a fundamental desire of mankind. A potential evolutionary roadmap for driver assistance systems is discussed. Emerging from systems based on proprioceptive sensors, such as ABS or ESC, we review the progress invented by the use of exteroceptive sensors such as radar, video, or lidar.

Driving Style Recognition Using a Smartphone as a Sensor Platform - To increase awareness and promote driver safety, we are proposing a novel system that uses Dynamic Time Warping (DTW) and smartphone based sensor-fusion to detect, recognize and record these actions without external processing. Our system differs from past driving pattern recognition research by fusing related inter-axial data from multiple sensors into a single classifier.

Platooning Control Using Visible Light Communications: A Feasibility Study. - This paper studies the applicability of visible light communications (VLC) system for information exchange between the platoon members. A complete VLC model is built enabling precise calculations of Bit-Error-Rate (BER) affected by inter-vehicle distance, background noise, and incidence angle and receiver electrical bandwidth. Based on our analytical model, the optical parameters suiting platooning application are defined. Finally, a SIMULINK model is developed to study the performances of a platooning exchange.

Experimental comparison of pulse-amplitude and spatial modulations for vehicle-to-vehicle visible light communication in platoon configurations. - In this paper, three forms of pulse amplitude modulations (PAM) are compared in a vehicular context: on-off keying (OOK), PAM-4 and generalized space-shift keying (GSSK). A prototype based on off-the-shelf light-emitting diodes (LED) headlamps is used for static tests in straight line configuration, with an inter-vehicle distance up to $30 \mathrm{~m}$, and curves of minimum radius $100 \mathrm{~m}$ and inter-vehicle distance of $10 \mathrm{~m}$. These tests show that OOK and GSSK are the most interesting modulations for highway platooning applications. Emergence of white-light LEDs allows synergy of lighting and broadcast/communication function in one optical source.

\section{METHODOLOGIES}

In the proposed system, the transmission of data from one vehicle to another is carry on in a very easier by Li-Fi technology by using led light. In this method, it will help us to avoid road accidents. In future the data transmission using Li-Fi technology will be promised to play a vital role in human's life.

Here in this proposed method, the problem arises in WiFi like the speed and the jamming are really take place when number of users increasing. But this traffic problem get reduces to a great number by using LI-Fi technology and this will proceed towards the cleaner, greener, safer and brighter future in this world without radio wave, because radio waves create a harmful effect for living thing, but Li-Fi is the optical wireless communication for data, audio and video streaming in LEDs. The ultrasonic sensor is used to measure the distance between the nearby vehicles and the vibration sensor is used to prevent the accidents. 
Vol. 8, Issue 2, February 2019

\section{Transmitter Section}

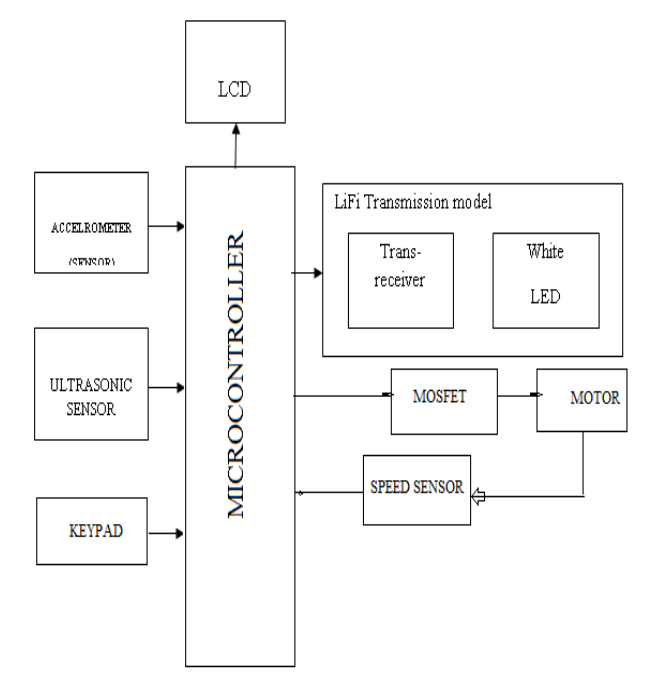

\section{Receiver Section}

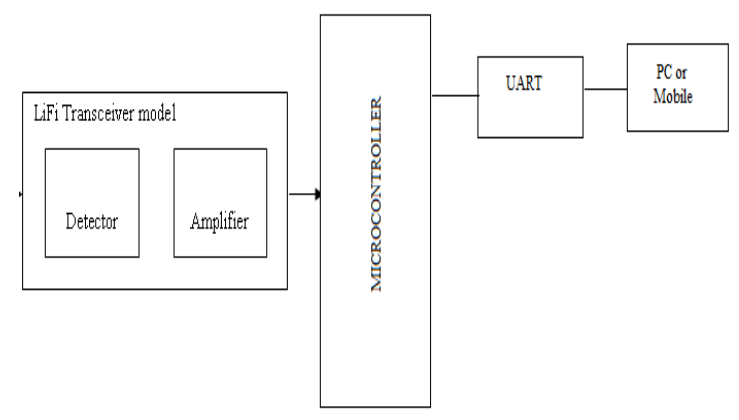

\subsection{COMPONENTS}

PIC MICROCONTROLER

PIC microcontroller is a microcontroller which uses the RISC architecture and it is the first microcontroller which uses the RISC architecture. RISC stands for Reduced Instruction Set Architecture. It allows simultaneous access to data and program memory and it is achieved due to separate bus for instruction and data.

\section{ULTRA SONIC SENSOR}

The ultra-sonic sensor used in this project is parallax PING. This sensor is mainly used in project application. This sensor consists of two parts namely the transmitter part and the receiver part. The transmitter part send the ultra-sonic waves and these waves get deflected and received in the receiver part. This finally calculates the time difference in the arrival of the waves, thus finding the distance between the vehicles.

\section{ACCELEROMETER}

The accelerometer used here is the ADXL335 which is a small, thin, low power complete 3-axis accelerometer. The output voltages are signal conditioning. The meter measures acceleration with a variation in the full-scale range between $+3 \mathrm{~g}$ to $3 \mathrm{~g}$. The static acceleration of gravity and dynamic acceleration resulting from the in tilt-sensing applications and motion respectively can be measured. Bandwidth of the accelerometer can be selected by the user using the $\mathrm{CX}$, $\mathrm{CY}$, and $\mathrm{CZ}$ capacitors namely $\mathrm{CX}, \mathrm{CY}$ and $\mathrm{CZ}$ which are placed at the XOUT, YOUT, and ZOUT pins.

\section{MOSFET DRIVER}

The MOSFET Driver used here is the IR2110/IR2113. This driver is more speed and have high voltage and has separate high and low side output channels. Both the Proprietary HVIC and latch immune CMOS technologies change monolithic construction. Logic inputs are compatible with standard CMOS output and it is down to 3.3V-3V logic. The output drivers encompasses a feature of high pulse current buffer stage that is intended for minimum driver cross-conduction. 


\title{
International Journal of Advanced Research in Computer and Communication Engineering
}

\author{
Vol. 8, Issue 2, February 2019
}

SOLAR PANNEL

The solar panel in this project act as a Li-Fi receiver. The solar panel consist of large number of photovoltaic cell, which are arranged in a linear manner. The panel captures the light emitted from the Li-Fi transmitter and then converts there data into the electrical signal, which is amplified by the amplifier.

$L C D$

The display unit is the LCD which is an $16 \times 2 \mathrm{LCD}$, which is a common type used. It has 16 rows and 2 columns [5×7] or [5×8] LCD dot matrices. The module we will use is number JHD162A. Available in 16 pin packages with features like contrast adjustment function, back light and each dot matrix has $5 \times 8$ dot resolution.

$L E D$

A Light-Emitting Diode (LED) is a diode, which is a light source of semiconductor material, which when current flows through it, emits light. When the holes and the electrons recombine it causes the emission of photons, which is the light. This effect is known as electroluminescence. The energy which is required for electrons to cross the band gap of the semiconductor band gap determines the light's colour.

POWER SUPPLY UNIT

The power supply unit in this project converts the incoming AC voltage of $220 \mathrm{v}$ into the required DC voltage. This unit consist of a rectifier circuit and a filter circuit. A regulator is used to distribute the necessary power to the different module.

\section{SERIAL USB TERMINAL}

'Serial USB Terminal' is a line-oriented terminal / console app for microcontrollers, Arduino and other devices with a serial / UART interface connected with a USB to serial converter to your android device.

\subsection{WORKING METHODOLOGIES}

The main component in this proposed model is the microcontroller. The microcontroller used here is PIC 16F877. All the components are connected to the microcontroller. There are three major units namely, the input unit, the output unit and the control unit. The input units are the ultrasonic sensor, the accelerometer. The ultrasonic sensor calculates the distance between the two vehicles and transfer these information to the microcontroller. The accelerometer calculates whether the driver turns the steering of the car left or right, and these data are also transferred to the micro controller. The control units are microcontroller and the three keys or switch which controls three action namely rising and decreasing the speed of the car and applying brakes. The DC motor here is prototyped as the wheels of the car. By using MOSFET driver, the speed of the motor is controlled by means of pulse width modulation. The output unit is LCD which displays the information of the transmitter vehicle. The data such as speed, distance between two vehicle and the steering direction of the car is also displayed.

\section{RESULTS}

Thus we had made a model for transmitting data by means of light between two vehicle and the information such as speed, distance between the two vehicle and the steering direction are made to transmit between these vehicles in every 5 seconds. An application namely "SERIAL USB TERMINAL" is used to display the transmitted information. The information is also displayed in the LCD screen and the motor is used as a prototype of a wheel.

\subsection{Transmitter part}

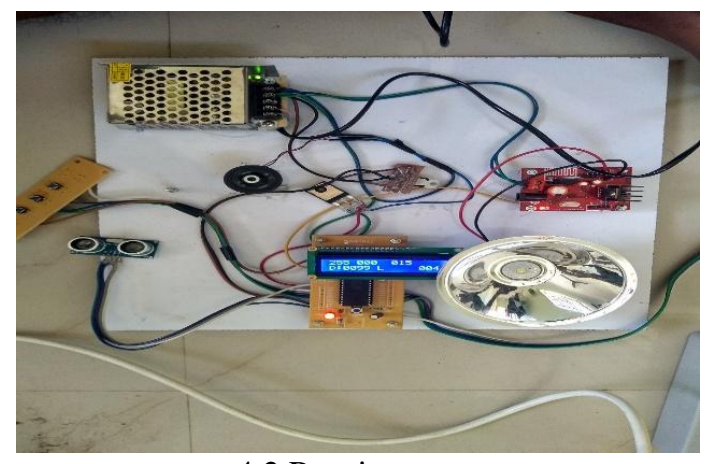

4.2 Receiver part 


\section{IJARCCE}

\section{International Journal of Advanced Research in Computer and Communication Engineering}

Vol. 8, Issue 2, February 2019

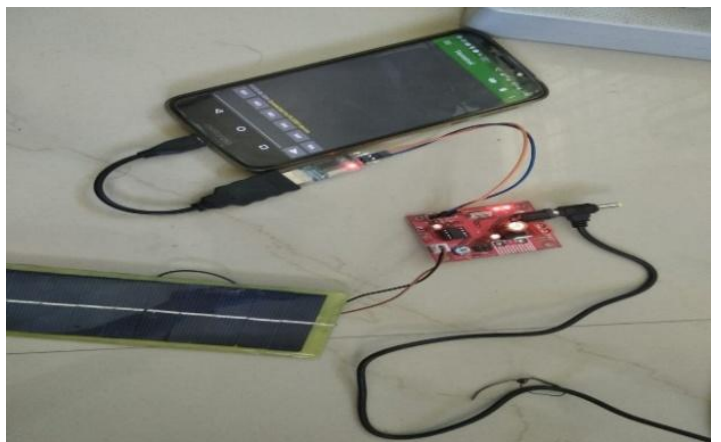

4.3 Experimental Output

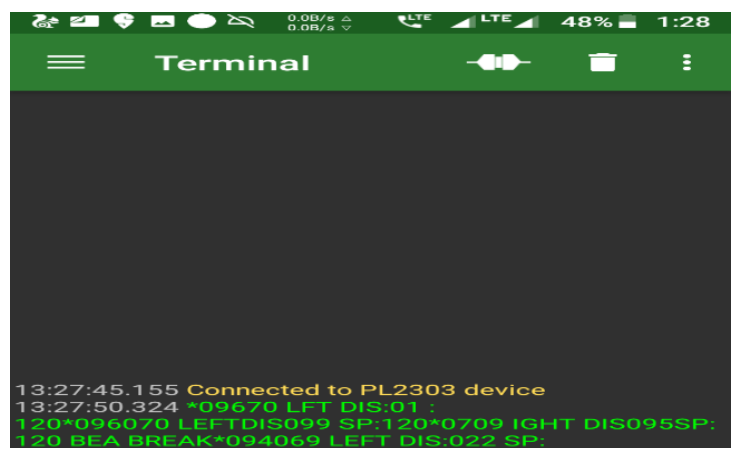

\section{CONCLUSION}

Thus we conclude that by implementing our model in real time we can avoid accidents and prevent life loss. By using this LI-FI technology the speed of the data transmission is increased and also the light rays do not have harmful effects in humans and is an easy medium of transmitting data.

\section{Future Scope}

1) We can also implement voice transmission methodology to inform the other vehicle about the status of our vehicle

2) This plays an important role in the ADAS

\section{Future Application of LI-FI}

- $\quad$ Education System

- Medical Application

- $\quad$ Cheaper Internet at Airports

- Underwater Applications

- Disaster Management

- $\quad$ Application in Sensitive Area

- $\quad$ Traffic Management

- Mobile Connectivity

\section{REFERENCES}

[1]. Hongyu Guan, Daniel Jutras, Zhuosha Guo (2018) "Secured and Green Data Processing and Transmission in a Human Vehicle Interaction ADAS System".

[2]. M Monisha, G Sudheendra (2017) "LIFI-Light Fidelity Technology"

[3]. Pooja, Saroj, Manisha (2015) "Advantage and Limitation of Radio over Fibre System"

[4]. Wen-long jin, cangy Kwan (2011) "A Smartphone based Inter-Vehicle Communication System” 